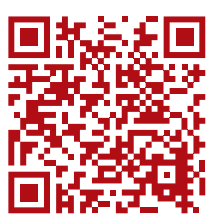

Palabras clave: Ética, cirugía plástica, belleza.

Keywords: Ethics, plastic surgery, beauty.

\footnotetext{
* Médico Residente de Cirugía General.

¥ Médico adscrito al

Servicio de Cirugía

General.
}

Hospital Universitario de Saltillo. Saltillo,

Coahuila de Zaragoza, México.

Recibido:

19 enero 2021

Aceptado:

19 julio 2021

\section{Consideraciones éticas en cirugía plástica}

\author{
Ethical considerations in plastic surgery \\ Dr. Gastón Domínguez-Saavedra,* Dr. Juan Miguel Hernández-Galván
}

\section{RESUMEN}

La cirugía plástica es una especialidad médico-quirúrgica que tiene entre sus funciones llevar al cuerpo a la normalidad anatómica y funcional por medio de intervenciones para reconstruir deformidades y corregir deficiencias funcionales. El hombre siempre ha sentido la necesidad de comprender su actuación según los cánones de belleza determinados por las condiciones del desarrollo social que son expresados de manera diferente en cada sociedad. La actividad estética y la conciencia del individuo están determinadas por el sistema de relaciones sociales y por valores culturales inherentes a la época y a la sociedad. La apariencia es un factor esencial en las interrelaciones humanas y la buena apariencia siempre ha estado asociada con el éxito profesional y social, por lo que los médicos no podemos olvidar su importancia en el equilibrio biopsicosocial del individuo; representa a su vez, la definición de salud que plantea la Organización Mundial de la Salud. El concepto de belleza existe desde la antigüedad y desde entonces se busca mantener la apariencia juvenil y la belleza. La correlación de los aspectos éticos de la cirugía plástica es muy amplia; debe tomarse en cuenta la «apariencia», que para algunos es la clave para escalar dentro de su ámbito profesional. Es aquí donde el cirujano plástico debe poner las pautas para no crear «estándares de belleza», sino ser una herramienta para aumentar la seguridad de los individuos, sin caer en cuestiones lucrativas y «comerciales», enfocándose en el bienestar físico y psicológico del paciente. En este artículo mencionamos algunos aspectos a considerar para su aplicación desde el punto de vista bioético, tomando en cuenta diversos factores que pueden determinar si los procedimientos son aptos y/o adecuados «para toda la población».

\section{ABSTRACT}

Plastic surgery is a medical-surgical specialty which has among its functions to bring the body to anatomical and functional normality through surgery to reconstruct deformities and correct functional deficiencies. Man has always felt the need to understand his performance according to the standards of beauty determined by the conditions of social development which are expressed differently in each society. The aesthetic activity and the conscience of the individual are determined by the system of social relations and by cultural values inherent to the period and to the society. Appearance is an essential factor in human and good appearance has always been related to professional and social success, which is why we cannot forget its importance in the bio-psycho-social balance of the individual; it represents, in turn, the definition of health established by the World Health Organization. The concept of beauty has existed since ancient times and, since then maintaining youthful appearance and beauty are sought after. The correlation of the ethical aspects of plastic surgery is very broad; «appearance» must be considered, which for some is the key to climbing their professional ladder. It is here where the plastic surgeon must set guidelines not to create «beauty standards», but to be a tool to increase the safety of individuals, without getting into lucrative and «commercial» issues, focusing on the physical and psychological well-being of the patient instead. In this article we mention some aspects to consider for their application from the bioethical point of view, taking into account various factors that can determine whether the procedures are suitable and/or adequate «for the entire population».

\section{INTRODUCCIÓN}

$\mathrm{L}$ a cirugía plástica comprende tanto la cirugía reconstructiva como la cirugía estética. La cirugía reconstructiva se reali- za sobre estructuras anormales del cuerpo causadas por defectos congénitos, anomalías del desarrollo, traumatismos, infecciones, tumores o enfermedades. La cirugía estética se realiza para restaurar la forma o dar nueva
Citar como: Domínguez-Saavedra G, Hernández-Galván JM. Consideraciones éticas en cirugía plástica. Cir Plast. 2021; 31 (2): 83-88. https://dx.doi.org/10.35366/102751 
forma a estructuras del cuerpo para mejorar su aspecto.

En la mitología grecorromana, la belleza despertaba admiración. Según el mito, la diosa griega Afrodita, también conocida en la cultura latina como Venus, causaba alborozo por donde pasaba por su incomparable belleza. En la cultura occidental, heredera de los valores grecorromanos, las mujeres son admiradas por su belleza, considerada el principal atributo de valoración femenina. Adornada e incentivada a permanecer siempre bella, la mujer aprende, desde niña, la importancia de la apariencia para mantener y conquistar posiciones ascendentes en la sociedad. La belleza la torna poderosa y deseada.

El término estética lo utilizó por primera vez Alexander Baumgarten en 1750, quien tomó la palabra griega aisthesis -que significa «sentido»- y definió la belleza como: «la percepción de la sabiduría que se adquiere a través de los sentidos»; y consecuentemente la estética como la «ciencia del conocimiento sensitivo». Pero Baumgarten la consideró una forma confusa e inferior del pensamiento. Kant, en 1790, reclamó el estatus de la estética a la par de la ética y la lógica o razón pura. Hoy, después de haber considerado la estética del hombre como lo hizo Baumgarten, nos unimos a Kant para reclamar sus derechos y defender su valor. ${ }^{1}$

Todavía en la infancia y especialmente en la adolescencia, las jóvenes se sienten muy incomodas con ciertas partes de su cuerpo que, real o ilusoriamente, les parecen que están fuera de los estándares sociales. El escrutinio se intensifica cuando la persona presenta deformidades congénitas o resultantes de traumas, lesiones y neoplasias. En las jóvenes, nariz y mamas son blancos preferenciales de esa búsqueda; además, otras partes del cuerpo y del rostro, como el vientre, las caderas y los pómulos, no escapan de un riguroso juicio.

La cirugía plástica se ha dividido desde un punto de vista práctico en dos campos de acción:

- Cirugía reconstructiva o reparadora, que incluye a la microcirugía, está enfocada a disimular y reconstruir los efectos destructivos de un accidente o trauma. La cirugía reconstructiva hace uso de técnicas de osteosíntesis, traslado de tejidos mediante colgajos y trasplantes autólogos de partes del cuerpo sanas a las afectadas. Persigue reconstruir un defecto dejado por un accidente, quemadura, malformación congénita, o a causa de la extirpación de una lesión maligna. La idea es restaurar una estructura o una función perdida.

- Cirugía estética o cosmética, este último término de poca aceptación en los colectivos profesionales, prefiriéndose el primero de ellos, es realizada con la finalidad de cambiar aquellas partes del cuerpo que no son satisfactorias para el paciente. Pretende corregir los cambios ocurridos en el desarrollo de los jóvenes, como orejas aladas, deformidades nasales, crecimiento exagerado de las mamas, o bien los estragos dejados por el paso del tiempo, como flaccidez facial y bolsas palpebrales. En muchos casos, sin embargo, hay razones médicas (mamoplastia de reducción cuando hay problemas ortopédicos en la columna lumbar, o la blefaroplastia en casos graves de blefarochalasis, que incluso impiden la visión).

A partir de la Primera Guerra Mundial, esta disciplina comenzó a definirse como especialidad quirúrgica y fue alrededor de los años 60 y los 70 que llegó a su edad de oro hasta nuestros días, gracias a la incorporación de nuevas técnicas y al perfeccionamiento de otras.

\section{ÉTICA EN LA CIRUGÍA PLÁSTICA}

Los valores son las apreciaciones más significativas del hombre, es decir, son las necesidades superiores convertidas en aspiraciones e ideales. De tal modo, responden a diferentes significados: económicos, políticos, sociales, culturales, estéticos, religiosos, científicos y morales. Constituyen el fundamento legítimo de los principios y las normas morales. Éstos surgen en la conciencia social y son el resultado de la relación valorativa del hombre con la realidad. La moralidad no es como muchos dicen, un mecanismo restrictivo de la conducta humana, sino un instrumento que orienta la capacidad creadora del hombre a través de sus valores. ${ }^{2}$ 
Sócrates, Platón y Aristóteles, explicaban la ética como el arte de vivir para lograr la felicidad. Este concepto se ha mantenido en la historia y en la actualidad podemos ampliar esta definición y decir que es la ciencia de la moral y las obligaciones del hombre, las reglas que rigen la conducta, el actuar manteniendo los valores culturales, al cual se le denomina ethos. ${ }^{3}$

El juramento hipocrático descansaba en los principios de «no dañar» y de «hacer el bien». Desde la antigüedad griega, con Aristóteles en el plano de la reflexión teórica acerca de la conducta humana, se ha intentado definir qué es el bien y qué es el mal. Pero esto no ha resultado fácil, pues cada una de las teorías éticas ha asumido diferentes posiciones frente a esta definición. Mientras para unos el bien está vinculado a la felicidad o bienestar de los hombres, para otros está relacionado con el grado de utilidad, o con el poder, o el placer. En esa época la ética médica estaba limitada a la relación entre el médico y el paciente. Además, la medicina se ejercía desde la posición del saber y el poder y esto traía consigo el paternalismo y el autoritarismo, dejando muy poco espacio al criterio del propio paciente para decidir sobre su propia salud y vida.

La ética médica se puede definir como el conjunto de reglas y principios que rigen la conducta profesional del médico y que implican una dedicación a valores más elevados que la ganancia financiera.

En nuestra sociedad, la ética profesional, además de ser respetuosa, debe ser beligerante en defensa de los pacientes y de sus derechos. La toma de decisiones siempre supone una elección entre alternativas diferentes, en las que siempre una será mejor (o menos mala) para el paciente que el resto. Por ello es imprescindible que los procedimientos y planteamientos sigan basándose en los principios de la bioética.

Por lo tanto, los principios básicos de la bioética deben ser tomados en cuenta en todo momento al tomar la decisión de proponer por parte del médico un determinado procedimiento:

- Beneficencia: favorecer a quienes son afectados por la acción cuando se trata de un servicio.
- No maleficencia: Primun non nocere. La prudencia es una cualidad que evita accidentes y errores, con lo cual adquiere la virtud ética de no ser maleficente.

- Autonomía: capacidad de decidir en cosa propia.

- Justicia: describe preferentemente las relaciones entre grupos sociales, enfatizando la equidad en la repartición de recursos y bienes considerados comunes.

En cuanto a la estética, desde el punto de vista filosófico, es la rama que estudia el significado de la belleza en general, la naturaleza del arte y la validez de los juicios sobre la creación artística, independientemente de la naturaleza de dicha obra de arte, que en el presente estudio será el ser humano, máxima expresión de La Creación. ${ }^{4}$

A menudo a los especialistas en cirugía plástica se les compara con los artistas, por trabajar de acuerdo con el sentido de lo bello. Pero entre ambos existe una gran diferencia: el artista crea obras de arte para que sean admiradas por el resto de los hombres; sin embargo, el profesional de la salud ayuda a corregir ciertos detalles anatómicos con el fin de satisfacer determinadas necesidades humanas. El cirujano se enfrenta con la motivación psicológica de la intervención quirúrgica deseada y con las repercusiones psíquicas que se originan como resultado de un defecto físico o malformación, o simplemente por una desproporción de algún elemento del cuerpo o las huellas del paso de los años.

En este sentido se debe distinguir los distintos aspectos psicológicos que afectan al paciente, de acuerdo con sus causas: malformaciones congénitas, malformaciones adquiridas, desviación del ideal de belleza (asociados o no al envejecimiento).

Está claro que las personas viven en sociedad relacionadas con otras personas y con el mundo que les rodea y es en esa interacción que el hombre conforma su esfera espiritual, dentro de la cual se destaca su componente psíquico, que ejerce notable influencia en el conjunto de relaciones sociales. Los rasgos característicos de la apariencia física pueden generar una serie de trastornos psicológicos, bien por la no adaptación del individuo a su 
grupo de relaciones o por sentirse rechazado por los demás, suscitando una depresión en la autoestima.

Al afectarse su esfera psíquica, puede llegar a sufrir un desequilibrio en su salud y recurrir a los servicios médicos con el objetivo de realizar las correcciones anatómicas pertinentes, que le llevaran a un alza en su autoestima y al restablecimiento de relaciones armónicas con el resto de la sociedad. Por eso, la belleza puede jugar un papel importante en el afán legítimamente humano de alcanzar la felicidad.

Todo esto se encuentra mediado por rasgos característicos de la personalidad, pues no todos los seres humanos recurren a cirugías plásticas, en tanto logran colocar en primer plano dentro de su autoestima otros elementos como la inteligencia bien desarrollada que lo haga destacarse por sobre todos los demás, aunque no sea bello.

El punto de partida para poder enunciar de manera general una teoría de la responsabilidad por actos médicos en cirugía plástica, debe encontrarse en una caracterización amplia de los actos médicos. Por tales se entienden el conjunto de actividades mediante las cuales los profesionales de la medicina producen en el cuerpo humano, directa o indirectamente, efectos destinados a prevenir, curar o aliviar las enfermedades, así como rehabilitar las funciones perdidas o alteradas y a promover el bienestar físico, psíquico o social de las personas. La meta más importante del ejercicio de la medicina es promover, mantener y recuperar la salud, definida por la OMS como «un estado completo de bienestar físico, mental y social y no sólo como la ausencia de enfermedad o de invalidez».

En el pasado la cirugía plástica se examinaba con una óptica que desdibujaba el sentido esencial de los propósitos que con ella se persiguen. Hay quienes afirman que no tiene finalidad curativa. Muchas veces se ha llegado al extremo de afirmar que se sale del campo de la medicina para entrar en el de la vanidad y la ilusión; sin embargo, tiene una función social absolutamente definida. En muchas ocasiones, una lesión en el rostro, una cicatriz, o una nariz deforme, produce al paciente serios traumas psicológicos que le impiden desarrollar una vida normal y feliz. Alguien ha dicho que la fealdad puede convertirse en una enfermedad psíquica. Gracias a la cirugía plástica pueden corregirse muchos defectos corporales, rejuvenecer físicamente a los pacientes, aplicarles injertos o trasplantes de piel cabelluda para calvicies, corregir las mamas hipertróficas o hipotróficas, resecar grandes panículos adiposos en casos de obesidad, corregir cicatrices, corregir arrugas de la cara y de las manos, inyectar grasa, etcétera.

Con cierta frecuencia, al margen del derecho, algunos cirujanos plásticos, frente a intervenciones con finalidad estética frustrada y seguidas de proceso judicial, han pretendido fundamentar el fin curativo del acto médico, argumentando la existencia de afecciones de orden psíquico, determinantes de complejos en el paciente. Ello puede ciertamente ocurrir en la práctica, pero al cirujano no le corresponde, en sentido estricto, hacer un diagnóstico psiquiátrico, porque podría argüirse en su contra fácilmente el cargo de impericia. Por consiguiente, es prudente que cuando el paciente manifieste sus complejos, el cirujano opte por una consulta especializada que le sirva de apoyo a la finalidad terapéutica, que busca con el procedimiento quirúrgico que considere útil para el caso. De ahí la importancia de la valoración integral del paciente antes de realizarse la cirugía. De no actuar así, es conveniente que se abstenga de invocar el fin curativo de su intervención y, mediante el consentimiento informado, exponga la característica de una actividad médica de medio y no de resultado. ${ }^{5}$

El deseo de aparecer normal o estéticamente aceptable es más antiguo que la cirugía plástica. La ética puritana, que dominaba hasta hace poco en nuestra cultura y que desaprobaba el narcisismo, se está derrumbando con rapidez. La popularidad creciente de esta disciplina ha creado, desafortunadamente, una atmosfera carnavalesca, evidente en las sociedades de consumo donde, a través de los mensajes publicitarios de los medios de difusión, se ha creado un patrón de belleza en la sociedad y muchas mujeres, si no cumplen con él, se sienten inconformes con ellas mismas.

Es recomendable establecer criterios claros para la selección de los pacientes que acuden a solicitar los servicios del cirujano plástico. El facultativo debe buscar los factores de perso- 
nalidad que acrecientan el deseo de mejorar físicamente. Una persona inteligente, educada, que sepa escuchar y entienda con claridad los pros y los contras, es un buen candidato. Individuos que presentan un problema físico objetivo acerca del cual tienen un interés razonable, pero no neurótico, son buenos candidatos. Personas cuyo trabajo les exige un aspecto de bienestar y dinamismo, y que deben competir con personas más jóvenes, también son buenos candidatos. ${ }^{6}$

En el mundo actual, la distribución de los especialistas en cirugía plástica es muy desigual. Como sucede con la riqueza, la proporción varía inmensamente entre cifras extremas. Cumple un papel central en la apariencia del individuo como ser biopsicosocial. La imagen tiene un gran significado dentro de las interacciones humanas, debido a que contribuye a sembrar en las personas nuevas esperanzas e ilusiones de llevar una vida sana, participativa y competitiva socialmente. ${ }^{7}$

En la sociedad actual, consumista, en que los medios de comunicación masiva tratan con mucha frecuencia los temas de cirugía estética, ya sea para promoverlos como lo más novedoso, o bien para dar a conocer malos resultados de ella, es muy fácil para los médicos caer en una cirugía estética contraria a la ética. ${ }^{2}$

Falta de profesionalismo y abusos publicitarios: con frecuencia se ven en revistas de actualidad y avisos publicitarios, en los que se promueve habitualmente por médicos no certificados en la especialidad, tratamientos de maravillosos resultados, sin ninguna evidencia científica, lo que lleva a muchos pacientes a consultar y a someterse a ellos, con resultados no deseados o francamente con malos resultados. En el caso de procedimientos, no se les informa de los productos utilizados ni de las dosis, mucho menos de las contraindicaciones de su uso ni de sus posibles complicaciones, ya que ello ahuyenta al paciente o más bien «al cliente». Es corriente escuchar en los relatos de estos pacientes cuando recurren a un especialista calificado para su opinión que hubo mucha falta de comunicación previa y muy poca responsabilidad posterior.

Falsas expectativas: es frecuente ofrecer tratamientos que aparecen como simples, rápidos, de excelentes resultados y sin compli- caciones, «cirugía exprés», que se promociona especialmente en pacientes extranjeros que necesariamente deben volver a sus países a los pocos días y cuya evolución posterior, con sus complicaciones y resultados deben ser asumidos por otro médico.

Incitación a la cirugía estética: promoviendo concursos con rifas de cirugías o procedimientos por personas ajenas a la medicina, que lo único que buscan es lucrar a través de las expectativas de los pacientes.

Finalmente, dentro del ejercicio profesional sanitario en todos los aspectos, en investigación clínica o no clínica, así como procedimientos quirúrgicos, debemos tener en cuenta los siguientes principios para salvaguardar la integridad de nuestros pacientes:

\section{PRINCIPIOS DE HELSINKI DE LA ASOCIACIÓN MÉDICA MUNDIAL}

Adoptada por la 18 a Asamblea Médica Mundial, Helsinki, Finlandia, junio de 1964 y enmendada por la 29a Asamblea Médica Mundial, Tokio, Japón, octubre de 1975; la 35ํㅡㄹ Asamblea Médica Mundial, Venecia, Italia, octubre de 1983 y la 41a Asamblea Médica Mundial, Hong Kong, septiembre de 1989.

Es misión del médico salvaguardar la salud de las personas. Su conocimiento y conciencia están dedicados al cumplimiento de esta misión. La Declaración de Ginebra de la Asociación Médica Mundial vincula al médico a la aseveración: «La salud de mi paciente será mi empeño principal», y el Código Internacional de Ética Médica declara que «cuando un médico proporcione una asistencia médica que pudiera tener un efecto de debilitamiento del estado físico y mental del paciente, el médico deberá actuar únicamente en interés del paciente». La finalidad de la investigación biomédica que implica a personas debe ser la de mejorar los procedimientos diagnósticos, terapéuticos y profilácticos y el conocimiento de la etiología y patogénesis de la enfermedad.

El principio que podemos adaptar dentro de los procedimientos estéticos y/o reconstructivos es el siguiente:

Debe respetarse siempre el derecho de las personas a salvaguardar su integridad. Deben 
adoptarse todas las precauciones necesarias para respetar la intimidad de las personas y reducir al mínimo el impacto del estudio sobre su integridad física y mental y su personalidad.

Otro punto importante es la proporcionalidad terapéutica, la cual indica lo siguiente:

En la medicina el principio de proporcionalidad terapéutica sostiene que existe la obligación moral de implementar sólo aquellas medidas terapéuticas que guarden relación de debida proporción entre los medios empleados y el resultado previsible, aplicado a la cirugía estética principalmente, solo ofrecer lo «necesario» al paciente sin caer en la «exageración» $y$ las falsas expectativas hacia el mismo. ${ }^{8}$

\section{CONCLUSIONES}

En las sociedades de consumo no todos los principios de la bioética se cumplen en la cirugía plástica estética, dado que es una especialidad a la cual sólo tienen acceso aquellas personas con un alto nivel adquisitivo, poniéndose de manifiesto la inequidad existente en dichas sociedades. Es muy importante que los médicos involucrados en la cirugía plástica en general y en la estética en particular, tengan muy claros sus objetivos, que son primero que nada el beneficio del paciente, objetivos que no pueden estar desvinculados de los principios éticos.

\section{REFERENCIAS}

1. Salazar R. Historia de la cirugía plástica. En: Coiffman F, ed. Cirugía plástica, reconstructiva y estética. Bogotá: Científicas y técnicas, S.A.; 1994. pp. 6-11.

2. Pere Serret E. Aspectos médicos y éticos en cirugía plástica reparadora y estética. Bioética \& Debat 2008; 52: 6-8.

3. Zis A. Fundamentos de la estética marxista. Moscú: Editorial Progreso; 1987. p. 5.

4. Piquero J, Piquero V. Ética en la estética y cosmética. Academia Biomédica Digital 2002; 13. Disponible en: http://vitae.ucv.ve/?module $=$ articulo $\&$ rv $=85 \& n=3582$

5. Sánchez RK, Alessandrini GR. Algunas consideraciones éticas sobre la cirugía plástica. Rev Cubana Cir 2007; $46(4)$

6. Prieto MC. En torno a la bioética. Organización Mundial de la Salud; 2003 [Acceso 13 de febrero de 2006].

7. Tamayo A. Responsabilidad médico - legal en cirugía plástica. En: Coiffman F, ed. Cirugía plástica, reconstructiva y estética. Bogotá: Científicas y técnicas, S.A.; 1994. pp. 78-83.

8. Aguirre-Gas HG. Principios éticos de la práctica médica. Cir Cir 2004; 72 (6): 17.

Conflicto de intereses: Los autores declaran no tener conflicto de intereses.

Financiamiento: Los autores no recibieron patrocinio para llevar a cabo este artículo.

\footnotetext{
Correspondencia:

Dr. Gastón Domínguez-Saavedra

Calzada Francisco I. Madero Núm. 1291,

Col. Centro, 25000, Saltillo,

Coahuila de Zaragoza, México.

E-mail: gaston_dot@hotmail.com
} 